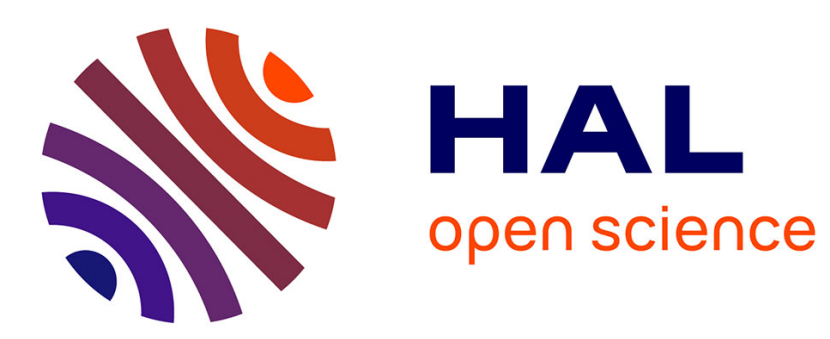

\title{
Robust transient stabilisation problem for a synchronous generator in a power network
}

Cristiano Verrelli, Gilney Damm

\section{To cite this version:}

Cristiano Verrelli, Gilney Damm. Robust transient stabilisation problem for a synchronous generator in a power network. International Journal of Control, 2010, 83 (4), pp.816-828. 10.1080/00207170903453209 . hal-00744974

\section{HAL Id: hal-00744974 \\ https://hal.science/hal-00744974}

Submitted on 18 Mar 2014

HAL is a multi-disciplinary open access archive for the deposit and dissemination of scientific research documents, whether they are published or not. The documents may come from teaching and research institutions in France or abroad, or from public or private research centers.
L'archive ouverte pluridisciplinaire HAL, est destinée au dépôt et à la diffusion de documents scientifiques de niveau recherche, publiés ou non, émanant des établissements d'enseignement et de recherche français ou étrangers, des laboratoires publics ou privés. 


\title{
Robust Transient Stabilization Problem for a Synchronous Generator in a Power Network
}

\author{
C. M. Verrelli ${ }^{a}$ and G. Damm ${ }^{b}$ \\ ${ }^{a}$ Department of Electronic Engineering, Tor Vergata University \\ Via del Politecnico 1, 00133 Rome, Italy \\ verrelli@ing. uniroma2.it \\ ${ }^{b}$ Laboratoire IBISC-CNRS, Université d'Evry Val d'Essonne \\ 40 rue du Pelvoux 91020 Evry Cedex, France \\ gilney.damm@ibisc.fr
}

\begin{abstract}
The robust transient stabilization problem (with stability proof) of a synchronous generator in an uncertain power network with transfer conductances is rigorously formulated and solved. The generator angular speed and electrical power are required to be kept close, when mechanical and electrical perturbations occur, to the synchronous speed and mechanical input power, respectively, while the generator terminal voltage is to be regulated, when perturbations are removed, to its pre-fault reference constant value. A robust adaptive nonlinear feedback control algorithm is designed on the basis of a third order model of the synchronous machine: only two system parameters (synchronous machine damping and inertia constants) along with upper and lower bounds on the remaining uncertain ones are supposed to be known. The conditions to be satisfied by the remote network dynamics for guaranteeing $\mathcal{L}_{2}$ and $\mathcal{L}_{\infty}$ robustness and asymptotic relative speed and voltage regulation to zero are weaker than those required by the single machine-infinite bus approximation: dynamic interactions between the local deviations of the generator states from the corresponding equilibrium values and the remote generators states are allowed.
\end{abstract}

Keywords. Robust nonlinear control, adaptive control, $\mathcal{L}_{2}$ and $\mathcal{L}_{\infty}$ disturbance attenuation, large-scale systems, power systems control.

\section{Introduction}

Power networks are among the most complex large-scale, interconnected nonlinear systems (see [1], [2] for the development of dynamical models of increasing complexity). They have continuously increased in size, power and number of components, but with a large acceleration in the last two decades. A rather difficult control problem, which attracted in the past the interest of the control community and has recently become a major concern as the dramatic 
blackouts in North America, Italy and Germany confirm, is represented by the power systems transient stabilization: dynamic nonlinear systems interconnected through a large-scale network are required to be controlled, on the basis of partial measurements of the local state variables only, in spite of time varying perturbations of almost all system parameters. In particular, the transient stabilization problem consists in the design of suitable excitation feedback controls such that: i) the angular speed and electrical power of each generator are kept close to the synchronous speed and mechanical input power, respectively, when mechanical and electrical perturbations, such as load shedding, generation tripping or short circuits, occur; ii) the terminal voltage of each generator is regulated to its pre-fault reference constant value when perturbations are removed. Decentralized linear controllers were first designed on the basis of linear approximations around operating conditions. This strategy was motivated by the fact that power systems were relatively small since production and consumption areas were not far. However, this situation has largely changed in the last two decades since the small power systems have been interconnected in order to increase performance and security: large amounts of electric power fluxes are transmitted from one region to another to cope with timevarying consumption and production levels. As a consequence, the overall system complexity has largely increased and the behaviour of such scattered plants has become unpredictable with disregarded nonlinear network phenomena arising: linear controllers may be no longer able to guarantee the power grid stability (see [3]) and to handle the severe disturbances and contingencies typically occurring in power networks. In the recent years, several nonlinear algorithms have been proposed for power systems control. For a particular power systems structure $\mathcal{M}$ consisting of a group of generators tied together by a strong network of transmission lines and linked to a single generator $g_{m}$ by a comparatively weak set of tie lines, the well-known single machine-infinite bus approximation, which models all the remaining network as a fixed voltage source and an impedance, is advantageous in the nonlinear control design: transient stabilization of the generator $g_{m}$ can be achieved, with a stability proof, even without requiring the knowledge of critical parameters ([3], [4], [5], [6], [7], [8], [9], [10], [11], [12]). In particular, $\mathcal{L}_{2}$ and $\mathcal{L}_{\infty}$ robustness and asymptotic relative speed regulation to zero are guaranteed in [8] despite uncertainties in all system parameters.

In this paper, we rigorously formulate and solve the transient stabilization problem (with stability proof) for the particular power systems structure $\mathcal{M}$ above considered. The single machine-infinite bus model, used in [3], [4], [5], [6], [7], [8], [9], [10], [11], [12] - which neglects the transient behaviour of the other generators as well as the interconnections between them, does not capture the typical multivariable nature with nonlinear complex coupling of power systems (see for instance the inter-area oscillations) and does not take into account the effect of the generator $g_{m}$ dynamics on the remote nework dynamics - is not used since interactions between the dynamics of the generator $g_{m}$ and the remote network machines are allowed: the transient behaviour of the remote generators states is allowed to depend on the local deviations of the generator states from the corresponding equilibrium values. Following the theoretical developments in [13], [14], [15] (even though they do not apply to the model considered in this paper in which an uncertain function multiplies the control input), a robust adaptive nonlinear feedback control is designed for the generator $g_{m}$ which does not assume the knowledge of the overall system parameters excepting for the machine damping and inertia constants. On the basis of upper and lower bounds on the uncertain model parameters, $\mathcal{L}_{2}$ and $\mathcal{L}_{\infty}$ robustness and transient stabilization are guaranteed under a set of assumptions on the network dynamics 
which are weaker than those required by the single machine-infinite bus approximation. The presented approach in conjunction with the special structure of power systems considered here allows us to rigorously formulate and solve the robust transient stabilization problem (with $\mathcal{L}_{2}$ and $\mathcal{L}_{\infty}$ robustness) of a synchronous generator in a power network in the presence of transfer conductances and uncertainties in almost all system parameters under possible dynamic interactions between the local deviations of the generator states from the corresponding equilibrium values and the remote generators states: this constitutes the main original contribution of the paper with respect to the results obtained in the literature for more general structures of power systems (see for instance [9], [16], [17], [18], [19], [20], [21], [22], [23], [24], [25]). Simulation results with reference to a general 3-machine, 9-buses power network show that the proposed robust nonlinear excitation control prevents each network machine from going out of step in the presence of electrical parameter perturbations and unmodelled dynamics and improves the performance with respect to the controller in [8] based on the single machine-infinite bus approximation.

\section{System dynamic model}

A power system consisting of $n$ generators interconnected through a transmission network is described by the $3 n$-order nonlinear models in [26], $1 \leq i \leq n$,

$$
\begin{aligned}
\dot{\delta}_{i} & =\omega_{i} \\
\dot{\omega}_{i} & =-\frac{D_{i}}{2 H_{i}} \omega_{i}+\frac{\omega_{0}}{2 H_{i}} P_{m i}-\frac{\omega_{0}}{2 H_{i}} P_{e i} \\
\dot{E}_{q i}^{\prime} & =\frac{k_{c i}}{T_{d 0 i}^{\prime}} u_{f i}-\frac{E_{q i}^{\prime}}{T_{d 0 i}^{\prime}}-\frac{\left(x_{d i}-x_{d i}^{\prime}\right)}{T_{d 0 i}^{\prime}} I_{d i}
\end{aligned}
$$

in which the first two equations represent the $i$-th generator mechanical dynamics involving the power angle $\delta_{i}(\mathrm{rad})$, the relative angular speed $\omega_{i}(\mathrm{rad} / \mathrm{s})$, the active electrical power $P_{e i}(\mathrm{p} . \mathrm{u}$.$) ,$ the mechanical input power $P_{m i}$ (p.u.), the synchronous speed $\omega_{0}(\mathrm{rad} / \mathrm{s})$, the damping constant $D_{i}$ (p.u.) (which is an often neglected non-negative parameter) and the inertia constant $H_{i}(\mathrm{~s})$, while the third equation constitutes the $i$-th generator electrical dynamics involving the transient EMF $E_{q i}^{\prime}$ (p.u.) in the quadrature axis, the input $u_{f i}$ (p.u.) to the thyristor amplifier, the gain $k_{c i}$ of the excitation amplifier, the direct axis transient open circuit time constant $T_{d 0 i}^{\prime}(\mathrm{s})$, the direct axis reactance $x_{d i}$ (p.u.) and the direct axis transient reactance $x_{d i}^{\prime}$ (p.u.). The $i$-th generator electrical equations are

$$
\begin{aligned}
P_{e i} & =E_{q i}^{\prime 2} G_{i i}+E_{q i}^{\prime} \sum_{j=1, j \neq i}^{n}\left[E_{q j}^{\prime} G_{i j} \cos \left(\delta_{i j}\right)+E_{q j}^{\prime} B_{i j} \sin \left(\delta_{i j}\right)\right]=v_{d i} I_{d i}+v_{q i} I_{q i} \\
Q_{e i} & =-E_{q i}^{\prime 2} B_{i i}+E_{q i}^{\prime} \sum_{j=1, j \neq i}^{n}\left[E_{q j}^{\prime} G_{i j} \sin \left(\delta_{i j}\right)-E_{q j}^{\prime} B_{i j} \cos \left(\delta_{i j}\right)\right]-x_{d i}^{\prime}\left(I_{d i}^{2}+I_{q i}^{2}\right) \\
& =v_{q i} I_{d i}-v_{d i} I_{q i} \\
I_{d i} & =-E_{q i}^{\prime} B_{i i}+\sum_{j=1, j \neq i}^{n}\left[E_{q j}^{\prime} G_{i j} \sin \left(\delta_{i j}\right)-E_{q j}^{\prime} B_{i j} \cos \left(\delta_{i j}\right)\right]
\end{aligned}
$$




$$
\begin{aligned}
I_{q i} & =E_{q i}^{\prime} G_{i i}+\sum_{j=1, j \neq i}^{n}\left[E_{q j}^{\prime} G_{i j} \cos \left(\delta_{i j}\right)+E_{q j}^{\prime} B_{i j} \sin \left(\delta_{i j}\right)\right] \\
\delta_{i j} & =\delta_{i}-\delta_{j} \\
V_{t i} & =\sqrt{\left(x_{d i}^{\prime} I_{q i}\right)^{2}+\left(E_{q i}^{\prime}-x_{d i}^{\prime} I_{d i}\right)^{2}}=\sqrt{v_{d i}^{2}+v_{q i}^{2}}
\end{aligned}
$$

in which (for the $i$-th generator): $Q_{e i}$ (p.u.) is the reactive electrical power, $I_{d i}$ (p.u.) is the direct axis current, $I_{q i}$ (p.u.) is the quadrature axis current, $V_{t i}$ (p.u.) is the terminal voltage with $(d, q)$-components $\left(v_{d i}, v_{q i}\right), G_{i j}$ (p.u.) and $B_{i j}$ (p.u.) are the $i$-th row and the $j$-th column element of nodal conductance and susceptance matrices, respectively, at the internal nodes after eliminating all physical buses, which depend on $x_{d i}^{\prime}$ (p.u.), on the transformer reactance $x_{T i}$ (p.u.), on the loads and on the transmission line reactance $x_{i j}$ (p.u.) between the $i$-th generator and the $j$-th generator. The nodal conductance and susceptance matrices represent the full power network of transmission lines and loads connecting the power generators: the network reduction of the load busses makes unsatisfactory the assumption of negligible transfer conductances (see [19]), which is typically used in power systems control design.

The pre-fault equilibrium, $1 \leq i \leq n$,

$$
\begin{aligned}
\delta_{i} & =\delta_{i s} \\
\omega_{i} & =0 \\
E_{q i}^{\prime} & =E_{q i 0}^{\prime}
\end{aligned}
$$

with

$$
P_{e i}=P_{m i}
$$

guarantees, when all model parameters take their unperturbed values, that the voltages $V_{t i}$, $1 \leq i \leq n$, are equal to their corresponding reference constant values $V_{t i}^{*}, 1 \leq i \leq n$.

In this paper, we study a particular structure of power systems, i.e., a group $G_{M}$ of $n-$ 1 generators, tied together by a strong network of transmission lines, which is linked to a generator $g_{m}$ (in the following referred to as $r$-th generator) by a comparatively weak set of tie lines. First note that the electrical power $P_{e i}$ affinely appears in the relative angular speed dynamics in (1): it represents an actually measured variable (thus available for feedback as a measured output) which is to be kept close to the mechanical input power during perturbations (thus to be controlled as a controlled output). Therefore it is advantageous to explicitly compute its dynamics in order to obtain a triangular structure for system (1) which is suitable for control design. Let us restrict the analysis to the $r$-th generator and let us compute $\left[G_{r j}\right.$ and $B_{r j}, 1 \leq j \leq n$, are assumed to be constant]

$$
\begin{aligned}
\dot{P}_{e r}= & \dot{E}_{q r}^{\prime}\left[I_{q r}+E_{q r}^{\prime} G_{r r}\right]+E_{q r}^{\prime} \sum_{j=1, j \neq r}^{n}\left[\dot{E}_{q j}^{\prime} G_{r j} \cos \left(\delta_{r j}\right)+\dot{E}_{q j}^{\prime} B_{r j} \sin \left(\delta_{r j}\right)\right] \\
& -E_{q r}^{\prime} \sum_{j=1, j \neq r}^{n}\left[E_{q j}^{\prime} G_{r j} \sin \left(\delta_{r j}\right)-E_{q j}^{\prime} B_{r j} \cos \left(\delta_{r j}\right)\right]\left[\omega_{r}-\omega_{j}\right] .
\end{aligned}
$$

We assume that there exist bounded connected open sets $\mathcal{D}_{\delta}$ and $\mathcal{D}_{p}$ such that $E_{q r}^{\prime}(0)>0$, $\delta_{r}(t) \in \mathcal{D}_{\delta}$ and $P_{e r}(t) \in \mathcal{D}_{p}$ imply $I_{q r}(t)>c_{I r}>0$, which describes the whole practical 
operating region of the generator (see [9]). The $r$-th generator third order dynamic model can be thus written as

$$
\begin{aligned}
\dot{\delta}_{r}= & \omega_{r} \\
\dot{\omega}_{r}= & -\frac{D_{r}}{2 H_{r}} \omega_{r}+\frac{\omega_{0}}{2 H_{r}} P_{m r}-\frac{\omega_{0}}{2 H_{r}} P_{e r} \\
\dot{P}_{e r}= & -\theta_{1 r} P_{e r}-\theta_{2 r} I_{d r} I_{q r}-\theta_{3 r} \frac{I_{d r} P_{e r}}{I_{q r}}-\theta_{4 r} \frac{P_{e r}^{2}}{I_{q r}^{2}}+\left[\frac{\theta_{5 r} I_{q r}^{2}+\theta_{6 r} P_{e r}}{I_{q r}}\right] u_{f r} \\
& -\left(Q_{e r}+B_{r r} \frac{P_{e r}^{2}}{I_{q r}^{2}}+x_{d r}^{\prime}\left(I_{d r}^{2}+I_{q r}^{2}\right)\right) \omega_{r}+\frac{P_{e r}}{I_{q r}} R_{r}
\end{aligned}
$$

in which $\delta_{r}, \omega_{r}, P_{e r}$ are the state variables, $u_{f r}$ is the control input, $\theta_{1 r}=\frac{1}{T_{d 0 r}^{\prime}}, \theta_{2 r}=\frac{\left(x_{d r}-x_{d r}^{\prime}\right)}{T_{d 0 r}^{\prime}}$, $\theta_{3 r}=\frac{G_{r r}\left(x_{d r}-x_{d r}^{\prime}\right)}{T_{d 0 r}^{\prime}}, \theta_{4 r}=\frac{G_{r r}}{T_{d 0 r}^{\prime}}, \theta_{5 r}=\frac{k_{c r}}{T_{d 0 r}^{\prime}}, \theta_{6 r}=\frac{G_{r r} k_{c r}}{T_{d 0 r}^{\prime}}$ and the term

$$
\begin{aligned}
R_{r}= & \sum_{j=1, j \neq r}^{n} \dot{\tilde{E}}_{q j}^{\prime}\left[G_{r j} \cos \left(\delta_{r j}\right)+B_{r j} \sin \left(\delta_{r j}\right)\right] \\
& +\sum_{j=1, j \neq r}^{n} \omega_{j}\left[E_{q j}^{\prime} G_{r j} \sin \left(\delta_{r j}\right)-E_{q j}^{\prime} B_{r j} \cos \left(\delta_{r j}\right)\right]
\end{aligned}
$$

represents the effect of the network remote dynamics (the group $G_{M}$ of generators) on the $r$-th generator, where $\tilde{E}_{q j}^{\prime}=E_{q j}^{\prime}-E_{q j 0}^{\prime}, 1 \leq j \leq n, j \neq r$. The expression of the function $R_{r}(t)$ will be crucial in the control design since it will allow us to rigorously formulate and solve the robust transient stabilization problem (with $\mathcal{L}_{2}$ and $\mathcal{L}_{\infty}$ robustness) of a synchronous generator in a power network characterized by the special structure in exam.

In the practice, the exact values of the model parameters are hard to obtain, and in particular $P_{m r}, G_{r r}, B_{r r}$ are lumped parameters which account for unmodelled dynamics such as turbine and load dynamics. Those parameters may undergo sudden on-line variations due to mechanical and electrical perturbations and faults. In the following, we will suppose the parameters $\omega_{0}, D_{r}, H_{r}$ to be known ${ }^{1}$ and will assume that: the uncertain piecewise continuous parameters $T_{d 0 r}^{\prime}(t), x_{d r}(t), x_{d r}^{\prime}(t), k_{c r}(t)$ and the uncertain constant parameter $G_{r r}$ are within the corresponding known positive bounds $\left(T_{d 0 r m}^{\prime}, T_{d 0 r M}^{\prime}\right),\left(x_{d r m}, x_{d r M}\right),\left(x_{d r m}^{\prime}, x_{d r M}^{\prime}\right),\left(k_{c r m}\right.$, $\left.k_{c r M}\right),\left(G_{r r m}, G_{r r M}\right)$; the uncertain constant parameter $B_{r r}$ is within the corresponding known bounds $\left(B_{r r m}, B_{r r M}\right)$; the mechanical input power $P_{m r}(t) \in \mathcal{D}_{p}$ is a class $\mathcal{C}^{1}$ function satisfying: $P_{m r M} \geq P_{m r}(t) \geq P_{m r m}$ and $\left|\dot{P}_{m r}(t)\right| \leq \dot{P}_{M r}$, with $P_{m r m}, P_{m r M}, \dot{P}_{M r}$ known positive reals. Physical considerations concerning transmission lines, loads and mechanical turbines make the above assumptions reasonable in the transient stabilization problem of power networks: upper and lower bounds on the equivalent conductances and susceptances can be computed, while the mechanical input power is usually the output of a slower first order dynamic system (controlled by a higher level control). On the other hand, the knowledge of bounds on the uncertain model parameters somehow restricts the on-line variations which can be tolerated by the controller and this is in line with the local result which will be obtained in the following.

\footnotetext{
${ }^{1}$ The parameters $D_{r}, H_{r}$ typically represent mechanical characteristics of the physical machine.
} 


\section{Control problem formulation}

A suitable control problem formulation is introduced in this section, which will allow us to solve the transient stabilization problem for the synchronous generator $g_{m}$ by quantitatively characterizing the robustness with respect to both permanent and vanishing model parameter perturbations. Let the pre-fault constant value for the power angle $\delta_{r}$ satisfying $\delta_{r s} \in \mathcal{D}_{\delta}$ and let $\theta_{i r m}, \theta_{i r M}$ be the known positive bounds on the uncertain parameters $\theta_{i r}(t), 1 \leq i \leq 6$, with $\hat{P}_{m r}(t)$ a suitable estimate of the uncertain mechanical power $P_{m r}$.

We will introduce in the following certain assumptions which are to be satisfied by the remaining part of the network in order to guarantee desired transient and asymptotic closed loop properties: they will impose conditions on the performance achieved by the controllers of the remote network generators (and in particular restrictions on the disturbance caused by remote network dynamics in response to the local deviations of the generator states from the corresponding equilibrium values) and will represent less restrictive conditions than those required by the single machine-infinite bus approximation in which no interaction between the local $r$-th generator deviations from $\left(\delta_{r}-\delta_{r s}, \omega_{r}, P_{e r}-P_{m r}\right)=0$ and the remote network dynamics is taken into account. They will allow dynamic (and not only static as in [16] and [22]) interactions between the local deviations of the generator states from the corresponding equilibrium values and the remote generators states: assumptions imposing only static interactions between the local deviations of the generator states from the corresponding equilibrium values and the remote generators states may not be able to comply with the typical instability phenomena in which the behaviour of generators in the network becomes oscillatory with increasing amplitudes. Let $\Sigma_{r}\left(y_{r 1}, y_{r 2}, y_{r 3}, R_{r}\right)$ be the system consisting of the $j$-th generators $(j \neq r, 1 \leq j \leq n)$ with inputs the $y_{r}(t)$ vector components $y_{r 1}(t), y_{r 2}(t), y_{r 3}(t)$ (i.e. the local $r$-th generator deviations from $\left.\left(\delta_{r}-\delta_{r s}, \omega_{r}, P_{e r}-P_{m r}\right)=0\right)$ and output the function $R_{r}(t)$. The subsequent assumption ii) will be satisfied if the controllers of the remote network generators make the system $\Sigma_{r}\left(y_{r 1}, y_{r 2}, y_{r 3}, R_{r}\right)$ Input to Output Stable with uniform gain functions $\bar{\varphi}_{\mu r}(\cdot), \bar{\varphi}_{\nu r}(\cdot), \bar{\varphi}_{\rho r}(\cdot)$, while the subsequent assumption iv) will be satisfied if the controllers of the remote network generators force the system $\Sigma_{r}\left(y_{r 1}, y_{r 2}, y_{r 3}, R_{r}\right)$ to have uniform finite $\mathcal{L}_{2}$ gains (recall that functions in $\mathcal{L}_{2}$ represent signals having finite energy over the infinite time interval $\left(t_{0},+\infty\right)$ and therefore the $\mathcal{L}_{2}$ gain can be interpreted as the ratio between the energies of the input and the output). Finally, the subsequent condition vi) will impose that, in the case of vanishing perturbations, the pre-fault state values for the remaining part of the network are asymptotically recovered provided that asymptotic regulation (S3) (for the $r$-th generator) is achieved. The transient stabilization problem addressed in this paper is rigorously formulated as follows.

Definition 1 (Transient Stabilizing Control): Assume that $(t \geq 0)$ :

i) for each $j \neq r, 1 \leq j \leq n$, the $j$-th generator power angle $\delta_{j}(t)$, the relative angular speed $\omega_{j}(t)$ and the quadrature axis transient $E M F E_{q j}^{\prime}(t)$ are piecewise differentiable functions of time $t$ and boundedness of the $r$-th generator variables $\delta_{r}(t), \omega_{r}(t), P_{e r}(t)$ implies boundedness of $E_{q j}^{\prime}(t)$; 
ii) there exist $\mu_{r}, \nu_{r}, \rho_{r}$ (unknown) non-negative reals, $\bar{\varphi}_{\mu r}(\cdot), \bar{\varphi}_{\nu r}(\cdot), \bar{\varphi}_{\rho r}(\cdot)$ known $\mathcal{K}_{\infty}$ functions ${ }^{2}$ and $g_{r}(t)$ (unknown) bounded non-negative real-valued function of time $t$ such that the following inequality:

$$
\begin{aligned}
\left|R_{r}(t)\right| \leq & \sup _{0 \leq \tau \leq t}\left\{g_{r}(\tau)\right\}+\mu_{r} \bar{\varphi}_{\mu r}\left(\max _{0 \leq \tau \leq t}\left\{\left|\delta_{r}(\tau)-\delta_{r s}\right|\right\}\right)+\nu_{r} \bar{\varphi}_{\nu r}\left(\max _{0 \leq \tau \leq t}\left\{\left|\omega_{r}(\tau)\right|\right\}\right) \\
& +\rho_{r} \bar{\varphi}_{\rho r}\left(\max _{0 \leq \tau \leq t}\left\{\left|P_{e r}(\tau)-P_{m r}(\tau)\right|\right\}\right)
\end{aligned}
$$

holds uniformly in $u_{f r}(\cdot)$.

Define $\left[\varepsilon_{j r}, \varepsilon_{x r}, \varepsilon_{B r}(1 \leq j \leq 6)\right.$ are positive reals]:

$$
\begin{aligned}
y_{r}(t)= & {\left[\delta_{r}(t)-\delta_{r s}, \omega_{r}(t), P_{e r}(t)-P_{m r}(t)\right]^{T} } \\
\xi_{r}(t)= & {\left[y_{r}(t)^{T}, P_{m r}(t)-\hat{P}_{m r}(t)\right]^{T} } \\
w_{d r}(t)= & {\left[\dot{P}_{m r}(t), \theta_{1 r M}-\theta_{1 r m}+\varepsilon_{1 r}, \theta_{2 r M}-\theta_{2 r m}+\varepsilon_{2 r}, \theta_{3 r M}-\theta_{3 r m}+\varepsilon_{3 r}, \theta_{4 r M}-\theta_{4 r m}+\varepsilon_{4 r},\right.} \\
& \max \left\{\theta_{5 r M}-\theta_{5 r m}+\varepsilon_{5 r}, \theta_{6 r M}-\theta_{6 r m}+\varepsilon_{6 r}\right\}, x_{d r M}^{\prime}-x_{d r m}^{\prime}+\varepsilon_{x r}, B_{r r M}-B_{r r m}+\varepsilon_{B r}, \\
& \left.\sup _{0 \leq \tau \leq t}\left\{g_{r}(\tau)\right\}, \mu_{r}, \nu_{r}, \rho_{r}\right]^{T} .
\end{aligned}
$$

A bounded piecewise continuous real-valued control law $u_{f r}(\cdot)$ is called a transient stabilizing control for the $r$-th generator if it guarantees the closed loop system to satisfy the following:

(S1) $\mathcal{L}_{\infty}$ disturbance attenuation property, i.e.

$$
\omega_{r}^{2}(t)+\left(P_{e r}(t)-P_{m r}(t)\right)^{2} \leq h_{1 r}\left(\xi_{r}(0)\right) e^{-c_{r} t}+\frac{1}{k_{r}} \gamma_{1 r}\left(\left\|w_{d r}(\cdot)\right\|_{\infty}\right)
$$

holds for all $t \geq 0$, where $h_{1 r}\left(\xi_{r}(0)\right) \geq 0, c_{r}>0$ and $\gamma_{1 r}(r)$ is a class $\mathcal{K}_{\infty}$ function;

(S2) $\mathcal{L}_{2}$ disturbance attenuation property, i.e.

$$
\int_{0}^{T}\left[\omega_{r}^{2}(\tau)+\left(P_{e r}(\tau)-P_{m r}(\tau)\right)^{2}\right] d \tau \leq h_{2 r}\left(\xi_{r}(0)\right)+\frac{1}{k_{r}} \int_{0}^{T} \gamma_{2 r}\left(\left\|w_{d r}(\tau)\right\|\right) d \tau
$$

holds for any given $T>0$, where $h_{2 r}\left(\xi_{r}(0)\right) \geq 0$ and $\gamma_{2 r}(r)$ is a class $\mathcal{K}_{\infty}$ function.

According to the previous definition, the effect of model parameters variations from their unperturbed values is quantified by properties $(\mathrm{S} 1)-(\mathrm{S} 2)$, which give bounds on $\mathcal{L}_{\infty}$ and $\mathcal{L}_{2}$ regulation errors. While the formulation introduced in Definition 1 leads to a closed loop robustness characterization, the adaptive formulation introduced in the following definitions allows us to obtain asymptotic relative speed regulation to zero in the case of definitely constant

\footnotetext{
${ }^{2}$ The requirement that the $\mathcal{K}_{\infty}$ functions $\bar{\varphi}_{\mu r}(\cdot), \bar{\varphi}_{\nu r}(\cdot), \bar{\varphi}_{\rho r}(\cdot)$ are known, which makes them to play the role of design functions, somehow restricts the remote network disturbance effects tolerated by the resulting controller, in line with the local result we are going to obtain.
} 
permanent parameter perturbations and asymptotic voltage regulation in the case of vanishing parameter perturbations.

Definition 2 (Transient Adaptive Stabilizing Control): Assume that, in addition to assumptions i)-ii), there exist non-negative reals $t_{0}, M_{R r}, \gamma_{\delta r}, \gamma_{\omega r}$ and $\gamma_{p r}$ such that:

iii) the uncertain machine parameters $P_{m r}(t), T_{d 0 r}^{\prime}(t), x_{d r}(t), x_{d r}^{\prime}(t), k_{c r}(t)$ are constant for all $t \geq t_{0}$

iv) the following inequality:

$$
\begin{aligned}
\lim _{t \rightarrow+\infty} \int_{t_{0}}^{t} R_{r}^{2}(\tau) d \tau \leq & M_{R r}+\gamma_{\delta r} \lim _{t \rightarrow+\infty} \int_{t_{0}}^{t}\left[\delta_{r}(\tau)-\delta_{r s}\right]^{2} d \tau+\gamma_{\omega r} \lim _{t \rightarrow+\infty} \int_{t_{0}}^{t}\left[\omega_{r}(\tau)\right]^{2} d \tau \\
& +\gamma_{p r} \lim _{t \rightarrow+\infty} \int_{t_{0}}^{t}\left[P_{e r}(\tau)-P_{m r}(\tau)\right]^{2} d \tau
\end{aligned}
$$

holds uniformly in $u_{f r}(\cdot)$.

A transient stabilizing control $u_{f r}(\cdot)$ is called a transient adaptive stabilizing control for the $r$-th generator if it guarantees, the additional property:

(S3) asymptotic regulation, i.e.

$$
\lim _{t \rightarrow+\infty}\left\|\left[\delta_{r}(t)-\delta_{r s}, \omega_{r}(t), P_{e r}(t)-P_{m r}\right]\right\|=0 .
$$

Definition 3 (Adaptive Regulating Control): Assume that, in addition to assumptions i)-ii), iv)

$v)$ there exist a non-negative real $\tilde{t}_{0}$ such that all the model parameters take their unperturbed values (i.e. all the perturbations are removed) for all $t \geq \tilde{t}_{0}$;

vi) under the asymptotic regulation property (S3), for each $j \neq r$,

$$
\begin{aligned}
\lim _{t \rightarrow+\infty}\left[\delta_{j}(t)-\delta_{j s}\right] & =0 \\
\lim _{t \rightarrow+\infty}\left[\omega_{j}(t)\right] & =0 \\
\lim _{t \rightarrow+\infty}\left[E_{q j}^{\prime}(t)-E_{q j 0}^{\prime}\right] & =0 .
\end{aligned}
$$

A transient stabilizing control $u_{f r}(\cdot)$ is called an adaptive regulating control for the $r$-th generator if it guarantees, the additional property:

(S4) asymptotic voltage regulation, i.e.

$$
\lim _{t \rightarrow+\infty}\left[V_{t r}(t)-V_{t r}^{*}\right]=0 .
$$




\section{Nonlinear robust design and stability analysis}

By virtue of techniques similar to those used in [6], we design, in this section, a transient adaptive stabilizing control ${ }^{3}$ for the $r$-th generator according to Definitions 1 and 2 . The subsequent control design is motivated by the choice of the quadratic function $\mathcal{V}_{r}$ and $\mathcal{W}_{r}$ which will be introduced in the following. Define the power angle regulation and relative angular speed tracking errors

$$
\begin{aligned}
\tilde{\delta}_{r} & =\delta_{r}-\delta_{r s} \\
\tilde{\omega}_{r} & =\omega_{r}-\omega_{r}^{*}
\end{aligned}
$$

with $\delta_{r s}$ being the power angle constant reference value and $\omega_{r}^{*}$ being the relative angular speed time-varying reference signal

$$
\omega_{r}^{*}=-\frac{5}{4} k_{\delta r} \tilde{\delta}_{r}
$$

We design the active electrical power time-varying reference signal $P_{e r}^{*}$ as

$$
P_{e r}^{*}=\frac{2 H_{r}}{\omega_{0}}\left[\frac{5}{4} k_{\omega r} \tilde{\omega}_{r}+\frac{5}{4} k_{\delta r} \omega_{r}-\frac{D_{r}}{2 H_{r}} \omega_{r}^{*}+\tilde{\delta}_{r}+\frac{1}{k_{\omega p r}} \tilde{\omega}_{r}\right]+\hat{P}_{m r}
$$

which relies on the estimate $\hat{P}_{m r}$ of the uncertain mechanical power $P_{m r}$ satisfying the estimation law ${ }^{4}$

$$
\begin{aligned}
\hat{P}_{m r}= & \phi_{r}+\frac{2 H_{r}}{\omega_{0}}\left(\frac{5}{4} k_{p e r}+\frac{k_{r}}{4}+\frac{1}{k_{r}}+\frac{\omega_{0}^{2}}{16 H_{r}^{2}} k_{\omega p r}\right) \omega_{r} \\
\dot{\phi}_{r}= & \left(\frac{5}{4} k_{p e r}+\frac{k_{r}}{4}+\frac{1}{k_{r}}+\frac{\omega_{0}^{2}}{16 H_{r}^{2}} k_{\omega p r}\right)\left[-\phi_{r}+\frac{D_{r}}{\omega_{0}} \omega_{r}+P_{e r}\right. \\
& \left.-\frac{2 H_{r}}{\omega_{0}}\left(\frac{5}{4} k_{p e r}+\frac{k_{r}}{4}+\frac{1}{k_{r}}+\frac{\omega_{0}^{2}}{16 H_{r}^{2}} k_{\omega p r}\right) \omega_{r}\right] \\
P_{m r m} \leq & \hat{P}_{m r}(0) \leq P_{m r M}
\end{aligned}
$$

and introduce the active electrical power tracking error

$$
\tilde{P}_{e r}=P_{e r}-P_{e r}^{*} \text {. }
$$

\footnotetext{
${ }^{3}$ Note that the control techniques developed in [8], [13], [14], [15] do not apply to the model (3) due to the presence of the uncertain term $\left(\theta_{5 r} I_{q r}^{2}+\theta_{6 r} P_{e r}\right)$ multiplying the control input $u_{f r}$.

${ }^{4}$ Note that the dynamics of the relative angular speed $\omega_{r}$ can be rewritten in the more compact form

$$
\dot{\omega}_{r}=\varpi_{r}+\frac{\omega_{0}}{2 H_{r}} P_{m r}
$$
}

in which

$$
\varpi_{r}=-\frac{D_{r}}{2 H_{r}} \omega_{r}-\frac{\omega_{0}}{2 H_{r}} P_{e r}
$$

is available for feedback. 
We design the robust control law $u_{f r}$ with the robustifying terms $v_{r}, \pi_{r}$ as

$$
\begin{aligned}
u_{f r}= & \frac{I_{q r}}{\left(\hat{\theta}_{5 r} I_{q r}^{2}+\hat{\theta}_{6 r} P_{e r}\right)} v_{r}-\frac{k_{r} I_{q r}\left(2 I_{q r}^{4}+2 P_{e r}^{2}\right) v_{r}^{2} \tilde{P}_{e r}}{4\left(\hat{\theta}_{5 r} I_{q r}^{2}+\hat{\theta}_{6 r} P_{e r}\right)^{2}\left(\theta_{5 r m} I_{q r}^{2}+\theta_{6 r m} P_{e r}\right)} \\
v_{r}= & -\frac{5}{4} k_{p r} \tilde{P}_{e r}+\frac{\omega_{0}}{2 H_{r}} \tilde{\omega}_{r}+\frac{5 D_{r} k_{\delta r}}{4 \omega_{0}} \omega_{r}+\frac{2 H_{r}}{\omega_{0}} \omega_{r}+\hat{\theta}_{1 r} P_{e r}+\hat{\theta}_{2 r} I_{d r} I_{q r}+\hat{\theta}_{3 r} \frac{I_{d r} P_{e r}}{I_{q r}} \\
& +\hat{\theta}_{4 r} \frac{P_{e r}^{2}}{I_{q r}^{2}}+\hat{B}_{r r} \frac{P_{e r}^{2} \omega_{r}}{I_{q r}^{2}}+\hat{x}_{d r}^{\prime}\left(I_{d r}^{2}+I_{q r}^{2}\right) \omega_{r}+Q_{e r} \omega_{r}-\frac{5 k_{\delta r}}{4 \omega_{0}}\left[D_{r} \omega_{r}-\omega_{0}\left(\hat{P}_{m r}-P_{e r}\right)\right] \\
& -\frac{2 H_{r}}{\omega_{0}}\left(\frac{5}{4} k_{\omega r}+\frac{1}{k_{\omega p r}}\right)\left[\left(\frac{5}{4} k_{\omega r}+\frac{D_{r}}{2 H_{r}}\right) \tilde{\omega}_{r}+\tilde{\delta}_{r}+\frac{\omega_{0}}{2 H_{r}} \tilde{P}_{e r}+\frac{1}{k_{\omega p r}} \tilde{\omega}_{r}\right]+\pi_{r} \\
\pi_{r}= & -\frac{k_{R r}}{4} \frac{P_{e r}^{2}}{I_{q r}^{2}} \tilde{P}_{e r}-\frac{k_{r}}{4} \tilde{P}_{e r}\left[P_{e r}^{2}+I_{d r}^{2} I_{q r}^{2}+\frac{I_{d r}^{2} P_{e r}^{2}}{I_{q r}^{2}}+\frac{P_{e r}^{4}}{I_{q r}^{4}}\left(1+\omega_{r}^{2}\right)+\omega_{r}^{2}\left(I_{d r}^{2}+I_{q r}^{2}\right)^{2}\right] \\
& -\frac{k_{r}}{4}\left(\frac{5}{4} k_{\omega r}+\frac{5}{4} k_{\delta r}+\frac{5}{4} k_{p e r}+\frac{k_{r}}{4}+\frac{1}{k_{r}}+\frac{\omega_{0}^{2}}{16 H_{r}^{2}} k_{\omega p r}+\frac{1}{k_{\omega p r}}\right)^{2} \tilde{P}_{e r} \\
& -\frac{k_{r}}{4} \frac{P_{e r}^{2}}{I_{q r}^{2}} \tilde{P}_{e r}\left[\bar{\varphi}_{\mu r}^{2}\left(\max _{0 \leq \tau \leq t}\left\{\tilde{\delta}_{r}(\tau)\right\}\right)+\bar{\varphi}_{\nu r}^{2}\left(\max _{0 \leq \tau \leq t}\left\{\tilde{\omega}_{r}(\tau)\right\}+\frac{5}{4} k_{\delta r} \max _{0 \leq \tau \leq t}\left\{\tilde{\delta}_{r}(\tau)\right\}\right)\right. \\
& +\bar{\varphi}_{\rho r}^{2}\left(\max _{0 \leq \tau \leq t}\left\{\tilde{P}_{e r}(\tau)\right\}+P_{m r M}-P_{m r m}+\frac{\dot{P}_{M r}}{k_{p e r}}+\frac{2 H_{r}}{\omega_{0}}\left(\frac{5}{4} k_{\omega r}+\frac{1}{k_{\omega p r}}+\frac{5}{4} k_{\delta r}\right) \max _{0 \leq \tau \leq t}\left\{\tilde{\omega}_{r}(\tau)\right\}\right. \\
& \left.\left.+\left[\frac{2 H_{r}}{\omega_{0}}\left(1+\frac{25}{16} k_{\delta r}^{2}\right)+\frac{5 D_{r} k_{\delta r}}{4 \omega_{0}}\right] \max _{0 \leq \tau \leq t}\left\{\tilde{\delta}_{r}(\tau)\right\}\right)+1\right]
\end{aligned}
$$

which $^{5}$ rely on the estimates $\hat{\theta}_{j r}, \hat{x}_{d r}^{\prime}, \hat{B}_{r r}$ of the uncertain parameters $\theta_{j r}, x_{d r}^{\prime}, B_{r r}$ whose estimation laws $(1 \leq j \leq 6)$ [functions $\xi_{\theta_{j}}(t), \xi_{x}(t), \xi_{b}(t)$ are yet to be chosen]

$$
\begin{aligned}
& \dot{\hat{\theta}}_{1 r}=\operatorname{Proj}\left[\xi_{\theta_{j}}(t), \hat{\theta}_{j r}, \theta_{j r m}, \theta_{j r M}, \varepsilon_{j r}\right], \theta_{j r m} \leq \hat{\theta}_{j r}(0) \leq \theta_{j r M} \\
& \dot{\hat{x}}_{d r}^{\prime}=\operatorname{Proj}\left[\xi_{x}(t), \hat{x}_{d r}^{\prime}, x_{d r m}^{\prime}, x_{d r M}^{\prime}, \varepsilon_{x r}\right], \quad x_{d r m}^{\prime} \leq \hat{x}_{d r}^{\prime}(0) \leq x_{d r M}^{\prime} \\
& \dot{\hat{B}}_{r r}=\operatorname{Proj}\left[\xi_{b}(t), \hat{B}_{r r}, B_{r r m}, B_{r r M}, \varepsilon_{B r}\right], \quad B_{r r m} \leq \hat{B}_{r r}(0) \leq B_{r r M}
\end{aligned}
$$

will be designed by using the projection algorithm $\operatorname{Proj}\left[\zeta, \hat{z}_{r}, z_{r m}, z_{r M}, \varepsilon_{z r}\right]$ in $[8]\left[\left(z_{r m}-\varepsilon_{z r}\right)>\right.$ 0]

$$
\operatorname{Proj}\left[\zeta, \hat{z}_{r}\right]= \begin{cases}\zeta & \text { if } z_{r m} \leq \hat{z}_{r} \leq z_{r M} \\ \zeta & \text { if } \hat{z}_{r}<z_{r m} \text { and } \zeta \geq 0 \\ \zeta & \text { if } \hat{z}_{r}>z_{r M} \text { and } \zeta \leq 0 \\ \zeta\left[1-\frac{z_{r m}{ }^{2}-\hat{z}_{r}^{2}}{\left.z_{r m^{2}-\left(z_{r m}-\varepsilon_{z r}\right)^{2}}\right]}\right. & \text { if } \hat{z}_{r}<z_{r m} \text { and } \zeta<0 \\ \zeta\left[1-\frac{\hat{z}_{r}^{2}-z_{r M}{ }^{2}}{\left(z_{r M}+\varepsilon_{r r}\right)^{2}-z_{r M}{ }^{2}}\right] & \text { if } \hat{z}_{r}>z_{r M} \text { and } \zeta>0\end{cases}
$$

\footnotetext{
${ }^{5}$ Note that infinite memory is formally required by the controller (7) to compute the time function $\max _{0<\tau<t}\{f(\tau)\}$ with $f(\cdot): \mathbb{R}_{0}^{+} \rightarrow \mathbb{R}$ piecewise differentiable (see Section 5 for a finite memory implementation of the controller).
} 
whose properties are the following:

1. $z_{r m}-\varepsilon_{z r} \leq \hat{z}_{r}(t) \leq z_{r M}+\varepsilon_{z r}, \forall t \geq 0$

2. Proj $\left[\zeta, \hat{z}_{r}, \cdot, \cdot, \cdot\right]$ is Lipschitz continuous;

3. $\left|\operatorname{Proj}\left[\zeta, \hat{z}_{r}, z_{r m}, z_{r M}, \varepsilon_{z r}\right]\right| \leq|\zeta|$;

4. $\left(z_{r}-\hat{z}_{r}\right) \operatorname{Proj}\left[\zeta, \hat{z}_{r}, z_{r m}, z_{r M}, \varepsilon_{z r}\right] \geq\left(z_{r}-\hat{z}_{r}\right) \zeta$

[which hold provided that the uncertain constant $z_{r}$ and the initial condition $\hat{z}_{r}(0)$ belong to the compact set $\left.\left[z_{r m}, z_{r M}\right]\right]$. Define the estimation errors $(1 \leq j \leq 6)$

$$
\begin{aligned}
\tilde{\theta}_{j r} & =\theta_{j r}-\hat{\theta}_{j r} \\
\tilde{x}_{d r}^{\prime} & =x_{d r}^{\prime}-\hat{x}_{d r}^{\prime} \\
\tilde{B}_{r r} & =B_{r r}-\hat{B}_{r r}
\end{aligned}
$$

so that, on the basis of the $r$-th generator dynamics (3), we obtain the error dynamics

$$
\begin{aligned}
\dot{\tilde{\delta}}_{r}= & -\frac{5}{4} k_{\delta r} \tilde{\delta}_{r}+\tilde{\omega}_{r} \\
\dot{\tilde{\omega}}_{r}= & -\left(\frac{5}{4} k_{\omega r}+\frac{D_{r}}{2 H_{r}}\right) \tilde{\omega}_{r}-\tilde{\delta}_{r}+\frac{\omega_{0}}{2 H_{r}} \tilde{P}_{m r}-\frac{\omega_{0}}{2 H_{r}} \tilde{P}_{e r}-\frac{1}{k_{\omega p r}} \tilde{\omega}_{r} \\
\dot{\tilde{P}}_{e r}= & -\frac{5}{4} k_{p r} \tilde{P}_{e r}+\frac{\omega_{0}}{2 H_{r}} \tilde{\omega}_{r}-\tilde{\theta}_{1 r} P_{e r}-\tilde{\theta}_{2 r} I_{d r} I_{q r}-\tilde{\theta}_{3 r} \frac{I_{d r} P_{e r}}{I_{q r}}-\tilde{x}_{d r}^{\prime}\left(I_{d r}^{2}+I_{q r}^{2}\right) \omega_{r} \\
& -\tilde{\theta}_{4 r} \frac{P_{e r}^{2}}{I_{q r}^{2}}+\tilde{\theta}_{5 r} \frac{I_{q r}^{2} v_{r}}{\left(\hat{\theta}_{5 r} I_{q r}^{2}+\hat{\theta}_{6 r} P_{e r}\right)}+\tilde{\theta}_{6 r} \frac{P_{e r} v_{r}}{\left(\hat{\theta}_{5 r} I_{q r}^{2}+\hat{\theta}_{6 r} P_{e r}\right)}-\tilde{B}_{r r} \frac{P_{e r}^{2} \omega_{r}}{I_{q r}^{2}} \\
& -\frac{k_{r}}{4} \frac{\left(2 I_{q r}^{4}+2 P_{e r}^{2}\right)\left(\theta_{5 r} I_{q r}^{2}+\theta_{6 r} P_{e r}\right) v_{r}^{2} \tilde{P}_{e r}}{\left(\hat{\theta}_{5 r} I_{q r}^{2}+\hat{\theta}_{6 r} P_{e r}\right)^{2}\left(\theta_{5 r m} I_{q r}^{2}+\theta_{6 r m} P_{e r}\right)}+\frac{P_{e r}}{I_{q r}} R_{r}+\pi_{r} \\
& -\left(\frac{5}{4} k_{\omega r}+\frac{5}{4} k_{\delta r}+\frac{5}{4} k_{p e r}+\frac{k_{r}}{4}+\frac{1}{k_{r}}+\frac{\omega_{0}^{2}}{16 H_{r}^{2}} k_{\omega p r}+\frac{1}{k_{\omega p r}}\right) \tilde{P}_{m r} \\
\dot{\tilde{P}}_{m r}= & -\left(\frac{5}{4} k_{p e r}+\frac{k_{r}}{4}+\frac{1}{k_{r}}+\frac{\omega_{0}^{2}}{16 H_{r}^{2}} k_{\omega p r}\right) \tilde{P}_{m r}+\dot{P}_{m r} .
\end{aligned}
$$

Consider the quadratic function

$$
\mathcal{V}_{r}=\frac{1}{2}\left(\tilde{\delta}_{r}^{2}+\tilde{\omega}_{r}^{2}+\tilde{P}_{e r}^{2}+\tilde{P}_{m r}^{2}\right)
$$

and compute the time derivative of function $\mathcal{V}_{r}$ along the trajectories of the error system (8). By using property 1. of the projection algorithm and by completing the squares, we obtain

$$
\begin{aligned}
\dot{\mathcal{V}}_{r} \leq & -\frac{5}{4}\left(k_{\delta r} \tilde{\delta}_{r}^{2}+k_{\omega r} \tilde{\omega}_{r}^{2}+k_{p r} \tilde{P}_{e r}^{2}+k_{p e r} \tilde{P}_{m r}^{2}\right)+\frac{1}{k_{r}}\left[\dot{P}_{m r}^{2}+\tilde{\theta}_{1 r}^{2}+\tilde{\theta}_{2 r}^{2}+\tilde{\theta}_{3 r}^{2}+\tilde{\theta}_{4 r}^{2}\right. \\
& \left.+\max \left\{\tilde{\theta}_{5 r}^{2}, \tilde{\theta}_{6 r}^{2}\right\}+\tilde{x}_{d r}^{\prime 2}+\tilde{B}_{i r}^{2}+\sup _{0 \leq \tau \leq t}\left\{g_{r}(\tau)\right\}^{2}+\mu_{r}^{2}+\nu_{r}^{2}+\rho_{r}^{2}\right]
\end{aligned}
$$


so that, according to (4)-(5) and property 1. of the projection algorithm, we can establish that the robust adaptive nonlinear feedback control algorithm (4)-(7) is a transient stabilizing control for the $r$-th generator. Suppose that, in addition to assumptions i)-ii), assumptions iii)-iv) hold and consider the quadratic function

$$
\mathcal{W}_{r}=\mathcal{V}_{r}+\frac{1}{2} \sum_{j=1}^{6} \beta_{j r} \tilde{\theta}_{j r}^{2}+\frac{1}{2} \beta_{x r} \tilde{x}_{d r}^{\prime 2}+\frac{1}{2} \beta_{B r} \tilde{B}_{i r}^{2}
$$

whose time derivative along the trajectories of the error system (8), for all $t \geq t_{0}$, is given by

$$
\begin{aligned}
\dot{\mathcal{W}}_{r}= & -\frac{5}{4} k_{\delta r} \tilde{\delta}_{r}^{2}-\left(\frac{5}{4} k_{\omega r}+\frac{D_{r}}{2 H_{r}}\right) \tilde{\omega}_{r}^{2}+\frac{\omega_{0}}{2 H_{r}} \tilde{P}_{m r} \tilde{\omega}_{r}-\frac{1}{k_{\omega p r}} \tilde{\omega}_{r}^{2}-\frac{5}{4} k_{p r} \tilde{P}_{e r}^{2}+\pi_{r} \tilde{P}_{e r} \\
& -\frac{k_{r}}{4} \frac{\left(2 I_{q r}^{4}+2 P_{e r}^{2}\right)\left(\theta_{5 r} I_{q r}^{2}+\theta_{6 r} P_{e r}\right) v_{r}^{2} \tilde{P}_{e r}^{2}}{\left(\hat{\theta}_{5 r} I_{q r}^{2}+\hat{\theta}_{6 r} P_{e r}\right)^{2}\left(\theta_{5 r m} I_{q r}^{2}+\theta_{6 r m} P_{e r}\right)}+\frac{5}{I_{q r}} R_{r} \tilde{P}_{e r}-\left(\frac{5}{4} k_{\omega r}+\frac{5}{4} k_{\delta r}+\frac{5}{4} k_{p e r}\right. \\
& \left.+\frac{k_{r}}{4}+\frac{1}{k_{r}}+\frac{\omega_{0}^{2}}{16 H_{r}^{2}} k_{\omega p r}+\frac{1}{k_{\omega p r}}\right) \tilde{P}_{m r} \tilde{P}_{e r}-\left(\frac{5}{4} k_{p e r}+\frac{k_{r}}{4}+\frac{1}{k_{r}}+\frac{\omega_{0}^{2}}{16 H_{r}^{2}} k_{\omega p r}\right) \tilde{P}_{m r}^{2} \\
& +\dot{P}_{m r} \tilde{P}_{m r}-\tilde{\theta}_{1 r}\left[P_{e r} \tilde{P}_{e r}+\beta_{1 r} \dot{\hat{\theta}}_{1 r}\right]-\tilde{\theta}_{2 r}\left[I_{d r} I_{q r} \tilde{P}_{e r}+\beta_{2 r} \dot{\hat{\theta}}_{2 r}\right]-\tilde{\theta}_{3 r}\left[\frac{I_{d r} P_{e r} \tilde{P}_{e r}}{I_{q r}}+\beta_{3 r} \dot{\hat{\theta}}_{3 r}\right] \\
& -\tilde{x}_{d r}^{\prime}\left[\left(I_{d r}^{2}+I_{q r}^{2}\right) \omega_{r} \tilde{P}_{e r}+\beta_{x r} \dot{\hat{x}}_{d r}^{\prime}\right]-\tilde{\theta}_{4 r}\left[\frac{P_{e r}^{2} \tilde{P}_{e r}}{I_{q r}^{2}+\beta_{4 r}} \dot{\hat{\theta}}_{4 r}\right]-\tilde{B}_{r r}\left[\frac{P_{e r}^{2} \omega_{r} \tilde{P}_{e r}}{I_{q r}^{2}}+\beta_{B r} \dot{\hat{B}}_{r r}\right] \\
& +\tilde{\theta}_{5 r}\left[\frac{I_{q r}^{2} v_{r} \tilde{P}_{e r}}{\left(\hat{\theta}_{5 r} I_{q r}^{2}+\hat{\theta}_{6 r} P_{e r}\right)}-\beta_{5 r} \dot{\hat{\theta}}_{5 r}\right]+\tilde{\theta}_{6 r}\left[\frac{P_{e r} v_{r} \tilde{P}_{e r}}{\left(\hat{\theta}_{5 r} I_{q r}^{2}+\hat{\theta}_{6 r} P_{e r}\right)}-\beta_{6 r} \dot{\hat{\theta}}_{6 r}\right] .
\end{aligned}
$$

If we choose the yet to be defined functions $\xi_{\theta_{j}}(t), \xi_{x}(t), \xi_{b}(t)(1 \leq j \leq 6)$ so that

$$
\begin{aligned}
& \dot{\hat{\theta}}_{1 r}=\operatorname{Proj}\left[-\frac{P_{e r} \tilde{P}_{e r}}{\beta_{1 r}}, \hat{\theta}_{1 r}, \theta_{1 r m}, \theta_{1 r M}, \varepsilon_{1 r}\right], \theta_{1 r m} \leq \hat{\theta}_{1 r}(0) \leq \theta_{1 r M} \\
& \dot{\hat{\theta}}_{2 r}=\operatorname{Proj}\left[-\frac{I_{d r} I_{q r} \tilde{P}_{e r}}{\beta_{2 r}}, \hat{\theta}_{2 r}, \theta_{2 r m}, \theta_{2 r M}, \varepsilon_{2 r}\right], \theta_{2 r m} \leq \hat{\theta}_{2 r}(0) \leq \theta_{2 r M} \\
& \dot{\hat{\theta}}_{3 r}=\operatorname{Proj}\left[-\frac{I_{d r} P_{e r} \tilde{P}_{e r}}{I_{q r} \beta_{3 r}}, \hat{\theta}_{3 r}, \theta_{3 r m}, \theta_{3 r M}, \varepsilon_{3 r}\right], \theta_{3 r m} \leq \hat{\theta}_{3 r}(0) \leq \theta_{3 r M} \\
& \dot{\hat{\theta}}_{4 r}=\operatorname{Proj}\left[-\frac{P_{e r}^{2} \tilde{P}_{e r}}{I_{q r}^{2} \beta_{4 r}}, \hat{\theta}_{4 r}, \theta_{4 r m}, \theta_{4 r M}, \varepsilon_{4 r}\right], \theta_{4 r m} \leq \hat{\theta}_{4 r}(0) \leq \theta_{4 r M} \\
& \dot{\hat{\theta}}_{5 r}=\operatorname{Proj}\left[\frac{I_{q r}^{2} v_{r} \tilde{P}_{e r}}{\left(\hat{\theta}_{5 r} I_{q r}^{2}+\hat{\theta}_{6 r} P_{e r}\right) \beta_{5 r}}, \hat{\theta}_{5 r}, \theta_{5 r m}, \theta_{5 r M}, \varepsilon_{5 r}\right], \theta_{5 r m} \leq \hat{\theta}_{5 r}(0) \leq \theta_{5 r M} \\
& \dot{\hat{\theta}}_{6 r}=\operatorname{Proj}\left[\frac{P_{e r} v_{r} \tilde{P}_{e r}}{\left(\hat{\theta}_{5 r} I_{q r}^{2}+\hat{\theta}_{6 r} P_{e r}\right) \beta_{6 r}}, \hat{\theta}_{6 r}, \theta_{6 r m}, \theta_{6 r M}, \varepsilon_{6 r}\right], \theta_{6 r m} \leq \hat{\theta}_{6 r}(0) \leq \theta_{6 r M}
\end{aligned}
$$




$$
\begin{aligned}
& \dot{\hat{x}}_{d r}^{\prime}=\operatorname{Proj}\left[-\frac{\left(I_{d r}^{2}+I_{q r}^{2}\right) \omega_{r} \tilde{P}_{e r}}{\beta_{x r}}, \hat{x}_{d r}^{\prime}, x_{d r m}^{\prime}, x_{d r M}^{\prime}, \varepsilon_{x r}\right], \quad x_{d r m}^{\prime} \leq \hat{x}_{d r}^{\prime}(0) \leq x_{d r M}^{\prime} \\
& \dot{\hat{B}}_{r r}=\operatorname{Proj}\left[-\frac{P_{e r}^{2} \omega_{r} \tilde{P}_{e r}}{\beta_{B r} I_{q r}^{2}}, \hat{B}_{r r}, B_{r r m}, B_{r r M}, \varepsilon_{B r}\right], \quad B_{r r m} \leq \hat{B}_{r r}(0) \leq B_{r r M}
\end{aligned}
$$

then, by using property 4 . of the projection algorithm and by completing the squares, we obtain

$$
\dot{\mathcal{W}}_{r} \leq-\frac{5}{4} k_{\delta r} \tilde{\delta}_{r}^{2}-\frac{5}{4} k_{\omega r} \tilde{\omega}_{r}^{2}-\frac{5}{4} k_{p r} \tilde{P}_{e r}^{2}-\frac{5}{4} k_{p e r} \tilde{P}_{m r}^{2}+\frac{R_{r}(t)^{2}}{k_{R r}} .
$$

Since $\tilde{\delta}_{r}, \tilde{\omega}_{r}, \tilde{P}_{e r}, \tilde{P}_{m r}$ are bounded, according to (8) and assumption i), $\dot{\tilde{\delta}}_{r}, \dot{\tilde{\omega}}_{r}, \dot{\tilde{P}}_{e r}, \dot{\tilde{P}}_{m r}$ are bounded so that $\tilde{\delta}_{r}(t), \tilde{\omega}_{r}(t), \tilde{P}_{e r}(t), \tilde{P}_{m r}(t)$ are uniformly continuous for all $t \geq t_{0}$. On the other hand, by virtue of assumptions iii)-iv), if

$$
k_{R r}>\max \left\{\frac{4 \bar{\gamma}_{\delta r}}{k_{\delta r}}, \frac{4 \bar{\gamma}_{\omega r}}{k_{\omega r}}, \frac{16 \gamma_{p r}}{k_{p r}}, \frac{16 \gamma_{p r}}{k_{p e r}}\right\}
$$

with

$$
\begin{aligned}
& \bar{\gamma}_{\delta r}=\gamma_{\delta r}+\frac{25}{8} \gamma_{\omega r} k_{\delta r}^{2}+4\left[\frac{2 H_{r}}{\omega_{0}}\left(\frac{25}{16} k_{\delta r}^{2}+1\right)+\frac{5}{4} \frac{D_{r} k_{\delta r}}{\omega_{0}}\right]^{2} \gamma_{p r} \\
& \bar{\gamma}_{\omega r}=2 \gamma_{\omega r}+4\left[\frac{2 H_{r}}{\omega_{0}}\left(\frac{5}{4} k_{\omega r}+\frac{5}{4} k_{\delta r}+\frac{1}{k_{\omega p r}}\right)\right]^{2} \gamma_{p r}
\end{aligned}
$$

then, by integrating (10) we obtain

$$
\lim _{t \rightarrow+\infty} \int_{t_{0}}^{t}\left[k_{\delta r} \tilde{\delta}_{r}^{2}(\tau)+k_{\omega r} \tilde{\omega}_{r}^{2}(\tau)+k_{p r} \tilde{P}_{e r}^{2}(\tau)+k_{p e r} \tilde{P}_{m r}^{2}(\tau)\right] d \tau \leq \mathcal{W}_{r}\left(t_{0}\right)+\frac{M_{R r}}{k_{R r}}
$$

so that, according to (4)-(5) and Barbalat's Lemma, we can establish that the robust adaptive nonlinear feedback control algorithm (4)-(7), (9) is a transient adaptive stabilizing control for the $r$-th generator. Finally, from (2) it is straightforward to establish that, under the additional assumptions v) (implying iii)) and vi), asymptotic voltage regulation (S4) is achieved so that the robust adaptive nonlinear feedback control algorithm (4)-(7), (9) is an adaptive regulating control for the $r$-th generator. The result holds for any initial condition (of the $r$-th generator) and positive control parameter $k_{r}$ maintaining, according to $(\mathrm{S} 1), \delta_{r}(t) \in \mathcal{D}_{\delta}$ and $P_{e r}(t) \in \mathcal{D}_{p}$ (guaranteeing $I_{q r}(t)>c_{I r}>0$ ) for all $t \geq 0$.

The main result of this paper can be summarized in the following theorem, which somehow ${ }^{6}$ extends the recent theoretical contribution in [8].

Theorem: The robust adaptive nonlinear feedback control algorithm (4)-(7), (9) is:

- a transient stabilizing control for the $r$-th generator;

\footnotetext{
${ }^{6}$ Here the machine damping and inertia constants are assumed to be known.
} 
- a transient adaptive stabilizing and an adaptive regulating control for the $r$-th generator when (see (11))

$$
k_{R r}>\max \left\{\frac{4 \bar{\gamma}_{\delta r}}{k_{\delta r}}, \frac{4 \bar{\gamma}_{\omega r}}{k_{\omega r}}, \frac{16 \gamma_{p r}}{k_{p r}}, \frac{16 \gamma_{p r}}{k_{p e r}}\right\},
$$

for any initial condition (of the $r$-th generator) and positive control parameter $k_{r}$ maintaining, according to $(S 1), \delta_{r}(t) \in \mathcal{D}_{\delta}$ and $P_{e r}(t) \in \mathcal{D}_{p}$ (guaranteeing $I_{q r}(t)>c_{I r}>0$ so that singularities in the controller are avoided) for all $t \geq 0$.

The robust adaptive nonlinear feedback control algorithm (4)-(7), (9) relies on: the available signals $^{7} \delta_{r}(t), \omega_{r}(t),\left(I_{d r}(t), I_{q r}(t)\right)$ (depending on a common reference for measuring the machine rotor angle), $P_{e r}(t), Q_{e r}(t)$ (recall (2) and see for instance [16], [17], [22], [24]); the positive control parameters $k_{\delta r}, k_{\omega r}, k_{p r}, k_{p e r}, k_{\omega p r}, k_{r}, k_{R r}, \beta_{j r}, \beta_{x r}, \beta_{B r}, \varepsilon_{j r}, \varepsilon_{x r}, \varepsilon_{B r}$ $(1 \leq j \leq 6)$ whose role may be evaluated by examining both the closed loop error equations and the corresponding functions $\mathcal{V}_{r}$ and $\mathcal{W}_{r}$ with their time derivatives. The parameters $k_{\delta r}$, $k_{\omega r}, k_{p r}, k_{p e r}$ direct affect the dynamics of $\tilde{\delta}_{r}, \tilde{\omega}_{r}, \tilde{P}_{e r}$ and $\tilde{P}_{m r}$, respectively; the parameters $k_{\omega p r}, k_{r}, k_{R r}$ characterize the robustifying terms in (5), (6) and (7); the parameters $\beta_{j r}, \beta_{x r}$, $\beta_{B r}(1 \leq j \leq 6)$ are the adaptation gains for $\hat{\theta}_{j r}, \hat{x}_{d r}^{\prime}, \hat{B}_{r r}(1 \leq j \leq 6)$ and the larger they are chosen the slower the adaptations for $\hat{\theta}_{j r}, \hat{x}_{d r}^{\prime}, \hat{B}_{r r}(1 \leq j \leq 6)$ result.

\section{Simulation results}

In this section we illustrate the performance and the robustness of the feedback control algorithm (4)-(7), (9) in the presence of unmodelled dynamics: the proposed control is applied to each generator of the popular Western System Coordinating Council (WSCC) 3-machine, 9-bus system reported in [26] and [30] $\left[D_{i}=0,1 \leq i \leq 3\right]$ and described by the two-axis model ([26]) $[1 \leq i \leq 3]$

$$
\begin{aligned}
\dot{\delta}_{i} & =\omega_{i} \\
\dot{\omega}_{i} & =-\frac{D_{i}}{2 H_{i}} \omega_{i}+\frac{\omega_{0}}{2 H_{i}} P_{m i}-\frac{\omega_{0}}{2 H_{i}} P_{e i} \\
\dot{E}_{d i}^{\prime} & =-\frac{E_{d i}^{\prime}}{T_{q 0 i}^{\prime}}+\frac{\left(x_{q i}-x_{d i}^{\prime}\right)}{T_{q 0 i}^{\prime}} I_{q i} \\
\dot{E}_{q i}^{\prime} & =\frac{k_{c i}}{T_{d 0 i}^{\prime}} u_{f i}-\frac{E_{q i}^{\prime}}{T_{d 0 i}^{\prime}}-\frac{\left(x_{d i}-x_{d i}^{\prime}\right)}{T_{d 0 i}^{\prime}} I_{d i} \\
P_{e i} & =E_{q i}^{\prime} I_{q i}+E_{d i}^{\prime} I_{d i}=v_{d i} I_{d i}+v_{q i} I_{q i} \\
Q_{e i} & =E_{q i}^{\prime} I_{d i}-E_{d i}^{\prime} I_{q i}-x_{d i}^{\prime}\left(I_{d i}^{2}+I_{q i}^{2}\right)=v_{q i} I_{d i}-v_{d i} I_{q i} \\
I_{d i} & =\sum_{j=1}^{n}\left[E_{q j}^{\prime} G_{i j} \sin \left(\delta_{i j}\right)-E_{q j}^{\prime} B_{i j} \cos \left(\delta_{i j}\right)+E_{d j}^{\prime} G_{i j} \cos \left(\delta_{i j}\right)+E_{d j}^{\prime} B_{i j} \sin \left(\delta_{i j}\right)\right]
\end{aligned}
$$

${ }^{7}$ Methods for measuring the power angle $\delta_{r}$ can be found in [27], [28], [29]. 


$$
\begin{aligned}
I_{q i} & =\sum_{j=1}^{n}\left[E_{q j}^{\prime} G_{i j} \cos \left(\delta_{i j}\right)+E_{q j}^{\prime} B_{i j} \sin \left(\delta_{i j}\right)-E_{d j}^{\prime} G_{i j} \sin \left(\delta_{i j}\right)+E_{d j}^{\prime} B_{i j} \cos \left(\delta_{i j}\right)\right] \\
V_{t i} & =\sqrt{\left(E_{d i}^{\prime}+x_{d i}^{\prime} I_{q i}\right)^{2}+\left(E_{q i}^{\prime}-x_{d i}^{\prime} I_{d i}\right)^{2}}=\sqrt{v_{d i}^{2}+v_{q i}^{2}}
\end{aligned}
$$

from which the model (1) has been derived by neglecting the dynamics of the fast damperwinding $E_{d i}^{\prime}$ and by using the simplification $x_{q i}=x_{d i}^{\prime}$ with $x_{q i}$ (p.u.) being the quadrature axis reactance. The initial conditions for the state variables are computed by systematically solving the load-flow equations of the network and by computing the values of the algebraic variables. For $1 \leq i \leq 3$, the functions $\bar{\varphi}_{\mu i}(\cdot), \bar{\varphi}_{\nu i}(\cdot), \bar{\varphi}_{\rho i}(\cdot)$ are set equal to $\mathbb{I}(\cdot)_{[0,+\infty)}[\mathbb{I}(\cdot)$ is the identity function $]$ i.e. $\bar{\varphi}_{j i}(q)=q, j=\mu, \nu, \rho(q \in[0,+\infty))$, the control parameters are chosen as $k_{\delta i}=k_{\omega i}=k_{p e i}=k_{\omega p i}=1, k_{p i}=720, k_{i}=0.001, k_{R i}=0.1, \beta_{1 i}=\beta_{2 i}=\beta_{3 i}=\beta_{4 i}=$ $\beta_{5 i}=\beta_{6 i}=\beta_{x i}=\beta_{B i}=348000$ while the initial conditions for the parameter estimates are set equal to the corresponding unperturbed values. The time function $s(t)=\max _{0 \leq \tau \leq t}\{f(\tau)\}$ is implemented as

$$
\begin{aligned}
& s(t)=\left\{\begin{array}{ll}
f(t), & \text { if } f(t)>s(t-h) \\
s(t-h), & \text { otherwise }
\end{array}, t \geq h\right. \\
& s(0)=f(0)
\end{aligned}
$$

with $h$ the integration step and $t=l h\left(l \in \mathbb{N}_{0}\right)$. In order to avoid division by zero ${ }^{8}$, for $1 \leq i \leq 3, \eta\left(P_{e i}\right)$ and $\eta\left(I_{q i}\right)$ replace $P_{e i}$ and $I_{q i}$ in the control algorithm (4)-(7), (9), respectively, where

$$
\eta(\xi)= \begin{cases}\xi, & \text { if } \xi \geq 0.05 \\ 0.05, & \text { otherwise. }\end{cases}
$$

The goal of the simulation is to verify the effects of a three-phase fault occurring near bus 7 at the end of line 5-7 at $t=0.001 \mathrm{~s}$, which is cleared at $0.084 \mathrm{~s}$ by opening line 5-7 (circuit breakers reclose at $t=1 \mathrm{~s}$ ). Figure 1-2 show a satisfactory performance of the proposed control even with the physical saturations (which are hit several times according to Fig. 2(b)): despite the considered severe perturbation, synchronous speeds are quickly restored while regulation of both electrical power and terminal voltage is guaranteed. For comparison, the same simulation is performed by applying (to each generator of the network) the nonlinear robust adaptive control (with control parameters $k_{\delta}=0.1, k_{\omega}=3, k_{p}=120, k=0.001, \beta_{i}=0.0000001$, $1 \leq i \leq 7$ and initial conditions ${ }^{9} \hat{\theta}_{i}(0)=0 i=1,2,4,5, \hat{\theta}_{6}(0)=\frac{u_{f r 0} \sin \left(\delta_{r}(0)\right)}{\bar{v}_{2}(0)}, \hat{\theta}_{3}(0)=P_{m r}$, $\left.\hat{\theta}_{7}(0)=1\right)$

$$
\begin{aligned}
u_{f r} & =\frac{\hat{\theta}_{6}}{\sin \left(\delta_{r}\right)} \bar{v}_{2}-\frac{k}{4 \sin \left(\delta_{r}\right)} \tilde{P}_{e r} \bar{v}_{2}^{2} \\
P_{e r}^{*} & =\hat{\theta}_{7} \bar{v}_{1}+\frac{k}{4} \tilde{\omega}_{r} \bar{v}_{1}^{2}
\end{aligned}
$$

\footnotetext{
${ }^{8}$ Recall that the simulation is carried out by using the fourth order model and by applying the proposed controller to each generator of the network.

${ }^{9}$ We denote by $u_{f r 0}$ the nominal input value.
} 


$$
\begin{aligned}
& \bar{v}_{1}=-\hat{\theta}_{1} \omega_{r}+\hat{\theta}_{3}+k_{\delta} \omega_{r}+\frac{k}{4} \tilde{\omega}_{r} \omega_{r}^{2}+\frac{k}{4} \tilde{\omega}_{r}+k_{\omega} \tilde{\omega}_{r}+\tilde{\delta}_{r} \\
& \dot{\hat{\theta}}_{i}=\beta_{i} \operatorname{Proj}\left[\left(\phi_{2 i} \tilde{\omega}_{r}+\bar{\phi}_{3 i} \tilde{P}_{e r}\right), \hat{\theta}_{i}\right], \quad \hat{\theta}_{i}(0) \in\left[\theta_{i m}, \theta_{i M}\right], \quad i=1,3,4,5 \\
& \dot{\hat{\theta}}_{2}=\beta_{2} \operatorname{Proj}\left[\left(\bar{\phi}_{32} \tilde{P}_{e r}-\tilde{\omega}_{r} \tilde{P}_{e r}\right), \hat{\theta}_{i}\right], \quad \hat{\theta}_{2}(0) \in\left[\theta_{2 m}, \theta_{2 M}\right] \\
& \dot{\hat{\theta}}_{6}=\beta_{6} \operatorname{Proj}\left[-\bar{v}_{2} \tilde{P}_{e r}, \hat{\theta}_{i}\right], \quad \hat{\theta}_{6}(0) \in\left[\theta_{6 m}, \theta_{6 M}\right] \\
& \dot{\hat{\theta}}_{7}=\beta_{7} \operatorname{Proj}\left[\bar{v}_{1} \tilde{\omega}_{r}, \hat{\theta}_{i}\right], \quad \hat{\theta}_{7}(0) \in\left[\theta_{7 m}, \theta_{7 M}\right] \\
& \operatorname{Proj}\left[\zeta, \hat{\theta}_{i}\right]= \begin{cases}\zeta & \text { if } \theta_{i m} \leq \hat{\theta}_{i} \leq \theta_{i M} \\
\zeta & \text { if } \hat{\theta}_{i}<\theta_{i m} \text { and } \zeta \geq 0 \\
\zeta & \text { if } \hat{\theta}_{i}>\theta_{i M} \text { and } \zeta \leq 0 \\
\zeta\left[1-\frac{\theta_{i m^{2}}{ }^{2} \hat{\theta}_{i}^{2}}{\left.\theta_{i m^{2}-\left(\theta_{i m}-\rho_{i}\right)^{2}}\right]}\right. & \text { if } \hat{\theta}_{i}<\theta_{i m} \text { and } \zeta<0 \\
\zeta\left[1-\frac{\hat{\theta}_{i}^{2}-\theta_{i M}{ }^{2}}{\left(\theta_{i M}+\rho_{i}\right)^{2}-\theta_{i M}{ }^{2}}\right] & \text { if } \hat{\theta}_{i}>\theta_{i M} \text { and } \zeta>0\end{cases} \\
& \theta_{i m}\left(\theta_{i m}-\rho_{i}\right) \geq 0, \quad \theta_{i M}\left(\theta_{i M}+\rho_{i}\right) \geq 0 \\
& \bar{v}_{2}=-k_{p} \tilde{P}_{e r}+\hat{\theta}_{2} \tilde{\omega}_{r}-\frac{k}{4} \tilde{\omega}_{r}^{2} \tilde{P}_{e r}-\bar{\phi}_{30}-\sum_{i=1}^{5} \bar{\phi}_{3 i} \hat{\theta}_{i}-\frac{k}{4} \tilde{P}_{e r} \sum_{i=1}^{5} \bar{\phi}_{3 i}^{2} \\
& \phi_{21}=-\omega_{r}, \phi_{23}=1, \phi_{24}=\phi_{25}=0 \\
& \bar{\phi}_{30}=P_{e r} \omega_{r} \operatorname{cotg}\left(\delta_{r}\right)-k_{\delta}\left[\left(\frac{k}{4} \omega_{r}^{2}+\frac{k}{4}+k_{\omega}+\frac{1}{k_{\delta}}\right)\left(\hat{\theta}_{7}+\frac{k}{2} \tilde{\omega}_{r} \bar{v}_{1}\right)+\frac{k}{4} \bar{v}_{1}^{2}\right] \omega_{r} \\
& -\dot{\hat{\theta}}_{7} \bar{v}_{1}+\left(\dot{\hat{\theta}}_{1} \omega_{r}-\dot{\hat{\theta}}_{3}\right)\left(\hat{\theta}_{7}+\frac{k}{2} \tilde{\omega}_{r} \bar{v}_{1}\right) \\
& \bar{\phi}_{31}=\omega_{r} \bar{\phi}_{s}, \quad \bar{\phi}_{32}=P_{e r} \bar{\phi}_{s}, \quad \bar{\phi}_{33}=-\bar{\phi}_{s}, \quad \bar{\phi}_{34}=-P_{e r}, \quad \bar{\phi}_{35}=\omega_{r} \sin ^{2}\left(\delta_{r}\right) \\
& \bar{\phi}_{s}=\left[\left(\frac{k}{4} \omega_{r}^{2}+\frac{k}{4}+k_{\omega}+k_{\delta}-\hat{\theta}_{1}+\frac{k}{2} \tilde{\omega}_{r} \omega_{r}\right)\left(\hat{\theta}_{7}+\frac{k}{2} \tilde{\omega}_{r} \bar{v}_{1}\right)+\frac{k}{4} \bar{v}_{1}^{2}\right] \\
& \tilde{\delta}_{r}=\delta_{r}-\delta_{r s}, \quad \tilde{\omega}_{r}=\omega_{r}+k_{\delta} \tilde{\delta}_{r}, \quad \tilde{P}_{e r}=P_{e r}-P_{e r}^{*}
\end{aligned}
$$

designed in [8] on the basis of a (third order) single machine-infinite bus model. As illustrated by the simulation results reported in Figs. 3-4, neglecting the transient behaviour of the other generators and the interconnections between them may be critical for the control design in [8]: in the presence of the considered perturbation, synchronous speeds are not quickly restored while electrical power and terminal voltage regulations are not satisfactorily obtained.

\section{Conclusions}

The transient stabilization problem (with stability proof) has been rigorously formulated in Section 3 and solved in Section 4 for a particular power systems structure $\mathcal{M}$ consisting of a group of generators tied together by a strong network of transmission lines and linked to a single generator $g_{m}$ by a comparatively weak set of tie lines. A robust adaptive nonlinear feedback control (4)-(7), (9) has been designed for the generator $g_{m}$ which does not assume 

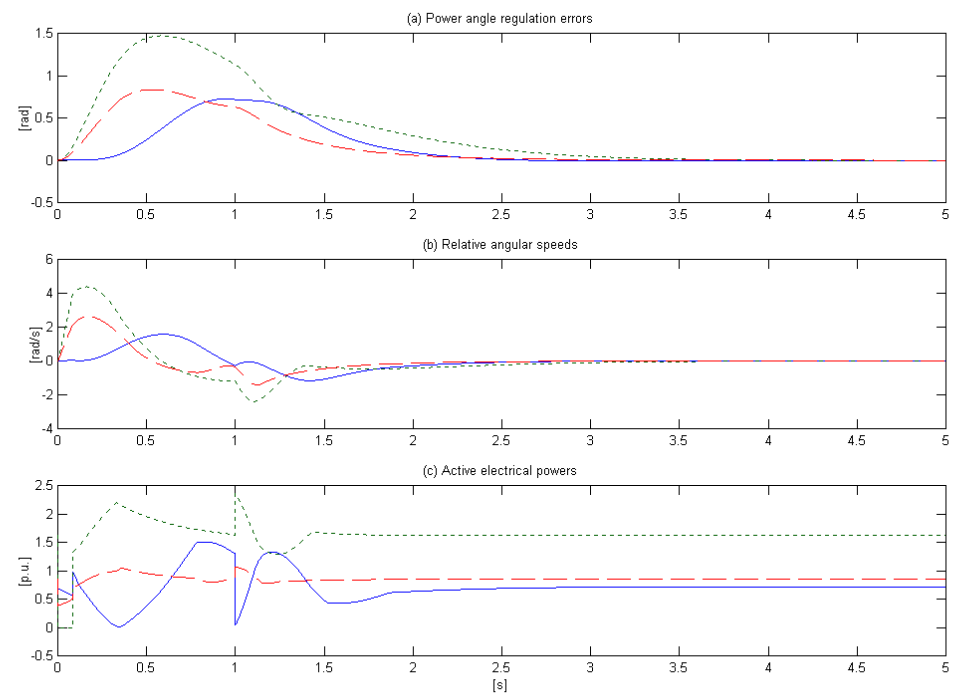

Figure 1: Proposed control algorithm [Generator 1 (solid), Generator 2 (dot), Generator 3 (dash)]: a) Power angle regulation errors $\delta_{i}-\delta_{i s}(1 \leq i \leq 3)$; b) Relative angular speeds $\omega_{i}$ $(1 \leq i \leq 3)$; c) Active electrical powers $P_{e i}(1 \leq i \leq 3)$.

the knowledge of the overall system parameters excepting for the machine damping and inertia constants: an innovative design technique has been used since available techniques developed in [8], [13], [14], [15] do not apply to the model (3). The proposed controller guarantees the $\mathcal{L}_{2}$ and $\mathcal{L}_{\infty}$ disturbance attenuation and asymptotic regulation properties (S1)-(S4) under assumptions i)-vi) on the network dynamics generalizing those required by the single machine-infinite bus approximation (which does not capture the typical multivariable nature with nonlinear complex coupling of power systems and does not take into account the effect of the generator $g_{m}$ dynamics on the remote nework dynamics) and allowing dynamic interactions between the local deviations of the generator states from the corresponding equilibrium values and the remote generators states (in order to comply with the typical instability phenomena in which the behaviour of generators in the network becomes oscillatory with increasing amplitudes). In our view the result of this paper constitutes a first step towards rigorous mathematical formulations and solutions to the multimachine transient stabilization problem.

\section{Acknowledgment}

The research was supported by the Italian Ministry of Education, University and Research and through a European Community Marie Curie Fellowship (in the framework of the CTS), c.n. HPMT-CT-2001-00278. The first author is indebted to Prof. R. Marino and P. Tomei for helpful discussions concerning this paper. 

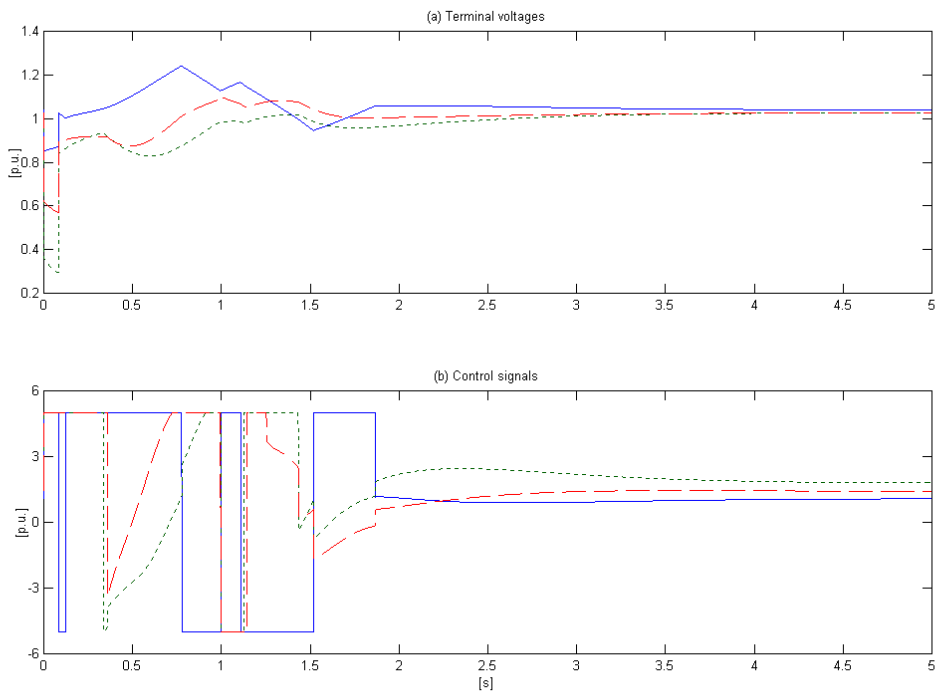

Figure 2: Proposed control algorithm [Generator 1 (solid), Generator 2 (dot), Generator 3 (dash)]: a) Terminal voltages $V_{t i}(1 \leq i \leq 3)$; b) Control signals $u_{f i}(1 \leq i \leq 3)$.

\section{References}

[1] Bergen AR, Vittal V. Power systems analysis. Prentice Hall: Upper Saddle River, 2000.

[2] Lu Q, Sun YZ, Mei S. Nonlinear control systems and power system dynamics. Kluwer: Boston, 2000.

[3] Guo Y, Hill DJ, Wang Y. Global transient stability and voltage regulation for power systems. IEEE Transactions on Power Systems 2001;16(4):678-688.

[4] Damm G, Marino R, Lamnabhi-Lagarrigue F. Adaptive nonlinear excitation control of synchronous generators. In Zinober A, Owens D (eds), Nonlinear and Adaptive Control, Springer Verlag: Berlin, 2003:65-76.

[5] Damm G, Marino R, Lamnabhi-Lagarrigue F. Adaptive nonlinear output feedback for transient stabilization and voltage regulation of power generators with unknown parameters. International Journal of Robust and Nonlinear Control 2004;14(9-10):833-855.

[6] Damm G, Marino R, Lamnabhi-Lagarrigue F, Verrelli CM. Transient stabilization and voltage regulation of a synchronous generator. In Lamnabhi-Lagarrigue $\mathrm{F}$, Laghrouche $\mathrm{S}$, Loria A, Panteley E (eds), Taming Heterogeneity and Complexity of Embedded Control, ISTE: London, 2006:211-225.

[7] Marino R, Damm G, Lamnabhi-Lagarrigue F. Adaptive nonlinear excitation control of synchronous generators with unknown mechanical power. In Isidori A, Lamnabhi-Lagarrigue 

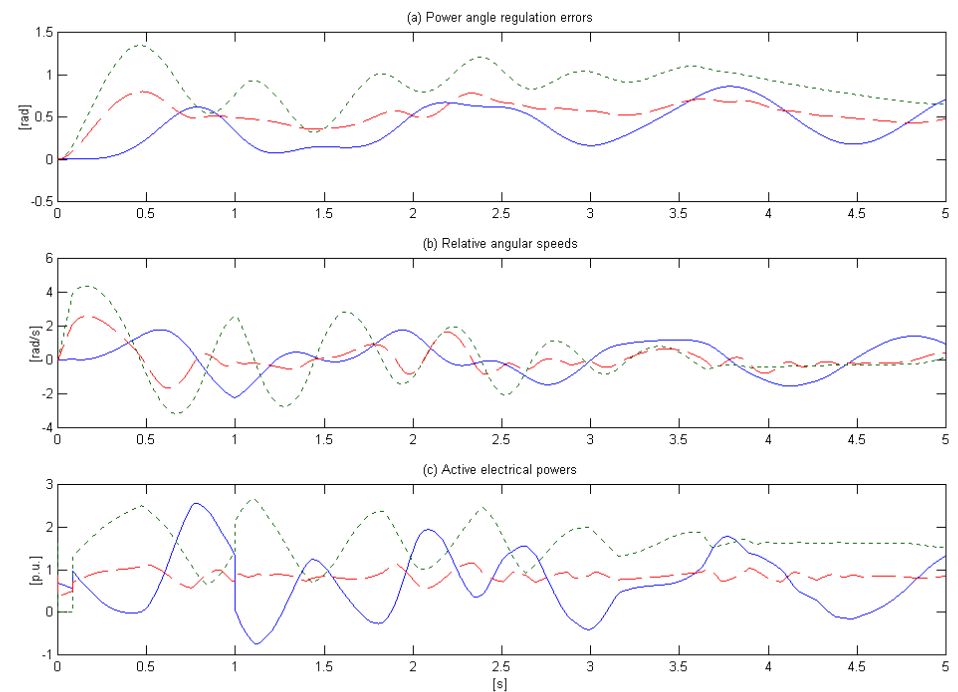

Figure 3: Robust adaptive control in [8] [Generator 1 (solid), Generator 2 (dot), Generator 3 (dash)]: a) Power angle regulation errors $\delta_{i}-\delta_{i s}(1 \leq i \leq 3)$; b) Relative angular speeds $\omega_{i}$ $(1 \leq i \leq 3)$; c) Active electrical powers $P_{e i}(1 \leq i \leq 3)$.

F, Respondek W (eds), Nonlinear Control in the Year 2000, Springer Verlag: London, $2001 ; 107-121$.

[8] Marino R, Shen T, Verrelli CM. Robust adaptive transient stabilization of a synchronous generator with parameter uncertainty. European Journal of Control 2006;12(2):135-148.

[9] Shen T, Mei S, Lu Q, Hu W, Tamura K. Adaptive nonlinear excitation control with $\mathcal{L}_{2}$ disturbance attenuation for power systems. Automatica 2003;39(1):81-89.

[10] Tan Y, Wang Y. Augmentation of transient stability using a superconducting coil and adaptive nonlinear control. IEEE Transactions on Power Systems 1998;13(2):361-366.

[11] Wang Y, Hill DJ. Robust nonlinear coordinated control of power systems. Automatica 1996;32(4):611-618.

[12] Wang Y, Hill DJ, Middleton RH, Gao L. Transient stabilization of power systems with an adaptive control law. Automatica 1994;30(9):1409-1413.

[13] Marino R, Tomei P. Robust adaptive state-feedback tracking control for nonlinear systems. IEEE Transactions on Automatic Control 1998;43(1):84-89.

[14] Marino R, Tomei P. Nonlinear output feedback tracking with almost disturbance decoupling. IEEE Transactions on Automatic Control 1999;44(1):18-28. 

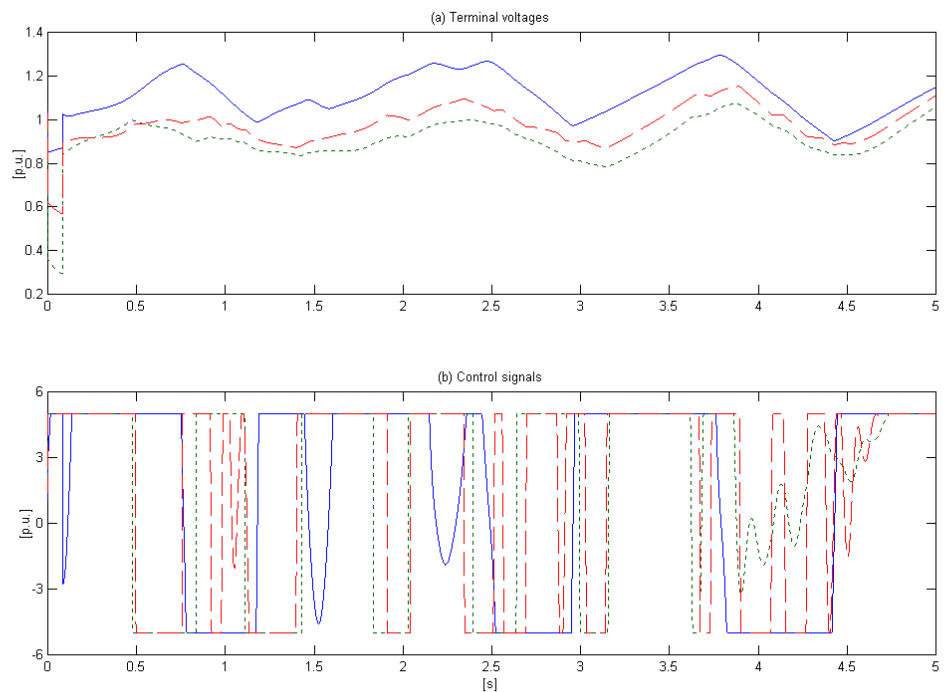

Figure 4: Robust adaptive control in [8] [Generator 1 (solid), Generator 2 (dot), Generator 3 (dash)]: a) Terminal voltages $V_{t i}(1 \leq i \leq 3)$; b) Control signals $u_{f i}(1 \leq i \leq 3)$.

[15] Shen T, Tamura K. Robust $H_{\infty}$ control of uncertain nonlinear systems via state feedback. IEEE Transactions on Automatic Control 1995;40(4):766-768.

[16] Guo Y, Hill DJ, Wang Y. Nonlinear decentralized control of large-scale power systems. Automatica 2000;36(9):1275-1289.

[17] Lu Q, Mei S, Hu W, Wu FF, Ni Y, Shen T. Nonlinear decentralized disturbance attenuation excitation control via new recursive design for multi-machine power systems. IEEE Transactions on Power Systems 2001;16(4):729-736.

[18] Machowski J, Robak S, Bialek JW, Bumby JR, Abi-Samra N. Decentralized stabilityenhancing control of synchronous generator. IEEE Transactions on Power Systems 2000;15(4):1336-1344.

[19] Ortega R, Galaz M, Astolfi A, Sun Y, Shen T. Transient stabilization of multimachine power systems with nontrivial transfer conductances. IEEE Transactions on Automatic Control 2005;50(1):60-75.

[20] Wang Y, Cheng D, Li C, Ge Y. Dissipative Hamiltonian realization and energy-based $\mathcal{L}_{2}$-disturbance attenuation control of multimachine power systems. IEEE Transactions on Automatic Control 2003;48(8):1428-1433.

[21] Wang Y, Cheng D, Liu Y, Li C. Adaptive $\mathcal{H}_{\infty}$ excitation control of multimachine power systems via the Hamiltonian function method. International Journal of Control 2004; $77(4): 336-350$. 
[22] Wang Y, Guo G, Hill DJ. Robust decentralized nonlinear controller design for multimachine power systems. Automatica 1997;33(9):1725-1733.

[23] Xi Z, Cheng D, Lu Q, Mei S. Nonlinear decentralized controller design for multimachine power systems using Hamiltonian function method. Automatica 2002; 38(3):527-534.

[24] Xi Z, Feng G, Cheng D, Lu Q. Nonlinear decentralized saturated controller design for power systems. IEEE Transactions on Control Systems Technology 2003;11(4):539-547.

[25] Zecevic AI, Neskovic G, Siljak DD. Robust decentralized exciter control with linear feedback. IEEE Transactions on Power Systems 2004;19(2):1096-1103.

[26] Sauer PW, Pai MA. Power system dynamics and stability. Prentice Hall: Upper Saddle River, 1998.

[27] Barrera-Cardiel E, Pastor-Gomez N. Microcontroller-based power-angle instrument for a power systems laboratory. IEEE Power Engineering Society Summer Meeting 1999;2:10081012.

[28] Chen Y, Zhang C, Hu Z, Wang X. A new approach to real time measurement of power angles of generators at different locations for stability control. Power Engineering Society Winter Meeting 2000;2:1237-1242.

[29] de Mello FP. Measurement of synchronous machine rotor angle from analysis of zero sequence harmonic components of machine terminal voltage. IEEE Transactions on Power Delivery 1994;9(4):1770-1777.

[30] Anderson PM, Fouad AA. Power system control and stability. Wiley-IEEE Press: New York, 2003. 